\title{
Estrategias de aprendizaje aplicadas a la asignatura motores de combustión interna para cumplimiento de logros de aprendizaje
}

Fecha de recepción : 2020-03-31 • Fecha de aceptación: 2020-06-04 • Fecha de publicación: 2020-09-10

\author{
Paco Ruben Alulema Ibarra ${ }^{1}$ \\ Pontificia Universidad Católica del Ecuador - Sede Ambato \\ pacoalulema68@gmail.com \\ https://orcid.org/0000-0001-8982-3983 \\ Pablo Israel Amancha Proaño² \\ Pontificia Universidad Católica del Ecuador - Sede Ambato \\ pamancha@pucesa.edu.ec \\ https://orcid.org/0000-0003-1502-6118
}

\section{Resumen}

La investigación tiene como objetivo establecer la relación existente entre las estrategias didácticas de aprendizaje y su influencia en los logros de aprendizaje de la asignatura de Motores de Combustión Interna $(\mathrm{MCl})$ de las Unidades Antonio Carrillo Moscoso y 12 de Noviembre del cantón Pillaro - Ecuador, con una muestra de 78 estudiantes y 4 docentes. En tal sentido, el estudio cuenta con un enfoque cualitativo y cuantitativo con un paradigma positivista; es decir, la metodología utilizada es de tipo mixto correlacional, pues se realiza una explicación de las estrategias docentes en el aula, y se determinan los logros de aprendizaje cumplidos, al comparar los resultados alcanzados por los estudiantes y lo esperado por las competencias curriculares. A su vez, se establece la asociación de las variables y se identifican cuáles son los factores que inciden en el desempeño de los alumnos. Las técnicas para la recolección de datos en este estudio son instrumentos validados y se realiza la prueba Chi Cuadrado. De este modo, se determinó que existe una correlación significativa en el uso de la estrategia con el logro de aprendizaje estudiantil. 
Palabras clave: estrategias didácticas, estrategias de aprendizaje, logros de aprendizaje, motores de combustión interna.

\section{Abstract}

The objective of the research is to establish the relationship between the didactic learning strategies and their influence on the learning achievements of the subject of Internal Combustion Engines (MCl) of the Antonio Carrillo Moscoso Units and November 12 of the Pillaro (Ecuador) canton with a sample 78 students and 4 teachers. In this sense, the study has a qualitative and quantitative approach with a positivist paradigm, that is, the methodology to be used is of a correlational type, since an explanation of the teaching strategies in the classroom is carried out, and the achievements of learning accomplished, by comparing the results achieved by students and what is expected by curricular competences. In turn, the association of the variables is established and the factors that affect student performance are identified. The techniques for data collection in this study are validated instruments and the Chi Square test is performed. Thus, it was determined that there is a significant correlation in the use of the strategy with the achievement of student learning.

Keywords: didactic strategies, learming strategies, learning achievements, internal combustion engine. 


\section{Introducción}

En la actualidad, la sociedad del conocimiento genera recursos y materiales didácticos al docente con la aplicación de Tecnologías de Información y Comunicación (TICs). Estos plantean un reto para el catedrático en la adaptación de estrategias y ambientes de aprendizaje a los nuevos paradigmas (Hernandez, 2017).

Este enfoque hacia las nuevas tecnologías ha sido marcado principalmente por la educación activa y colaborativa, que generan a su paso estrategias que describen las preferencias de los estudiantes durante las actividades de aprendizaje que involucran el incorporar y conciliar exitosamente información novedosa con la existente (Hoffman, Ledesma, y Liporace, 2017).

Dicho esto, se hace necesario desarrollar en el alumnado habilidades, formar valores y adquirir pericia para actuar de forma independiente. Por tal motivo, es ineludible la aplicación de métodos que propicien una instrucción intencional, auto-rregulada, reflexiva, consciente, que trace metas y objetivos propios (Montes De Oca \& Machado, 2011), donde existen tendencias para el uso de estrategias de aprendizaje en función de variables como género, tipo de carrera, antecedentes del estudio, estrato social y rendimiento académico (Visbal, Mendoza, y Díaz, 2017), las mismas que pueden mejorar con una serie de técnicas que consideren la personalidad del estudiante, los métodos que manejan, factores motivacionales e involucren al docente en el proceso; por ello se debe organizar grupos de estudiantes desde un punto de vista más cooperativo (Gillies, 2016).

Lo expuesto sugiere comprender que es necesario aplicar propuestas pedagógicas que favorezcan el aprendizaje autónomo, con una planificación y organización de actividades que desplieguen el uso estratégico de sus habilidades (García y Tejedor, 2017), sin dejar de reconocer el papel intrínseco que desarrolla el docente dentro del proceso catedrático.

Sin embargo, la enseñanza de contenidos prácticos se ha caracterizado por apoyarse en clases tradicionales, expositivas y monótonas; carentes de metodologías de educación activas y recursos tecnológicos que convierten al profesor en un transmisor de conocimientos, sin que exista una verificación del entendimiento asimilado por los alumnos en el momento donde, en general, los estudiantes no practican de manera intuitiva estrategias, las mismas que tienen influencia de los estilos educativos paternos (autoritarios, demo-cráticos y permisivos).

Esta es una debilidad que los alumnos de tercer año de bachillerato de la carrera de Electromecánica Automotriz, perteneciente a las Unidades Educativas Antonio Carrillo Moscoso y 12 de Noviembre del Cantón Píllaro presentan en su proceso de aprendizaje; por lo que surge la necesidad de relacionar las estrategias didácticas para que se pueda aplicar en las actividades cotidianas de la profesión, por lo que dicha fusión realizada de manera equilibrada podría dar solución a las necesidades actuales a través de un diagnóstico y un análisis exacto de la información recolectada.

El avance de la tecnología en los últimos años ha generado el impulso necesario para buscar nuevas alternativas innovadoras en el proceso de enseñanza-aprendizaje, desarrollando en los 
estudiantes nuevas habilidades y destrezas mediante actividades que mejoren su adquisición de conocimientos.

Según la conceptualización expuestas por Flores et al. (2017), las estrategias didácticas se interpretan como procedimientos y recursos que utiliza el docente para promover aprendizajes significativos que facilitan intencionalmente el procesamiento de los nuevos conocimientos, de manera profunda y consciente. Una reseña señala como técnicas que se emplean para manejar de la manera mas eficiente y sistemática el proceso de enseñanza-aprendizaje (Tabla 1).

Por otra parte están las estrategias de aprendizaje, las cuales Flores et al. (2017) explica que son un conjunto de acciones que se realizan para lograr un objetivo de instrucción con la aplicación de pasos cognitivos que identifican tanto habilidades y capacidades, así como métodos y técnicas en el estudio, tal como la capacidad de reflexionar la forma en que se aprende actuar, en consecuencia, regulando el autoaprendizaje mediante el uso de estrategias flexibles y apropiadas que se transfieren y adoptan a nuevas situaciones.

Tabla 1.

Tipos de estrategias didácticas

\begin{tabular}{ll}
\hline Tipo & Características \\
\hline Enseñanza & $\begin{array}{l}\text { Son planteadas por el docente y } \\
\text { proporcionadas al estudiante para } \\
\text { facilitar un procesamiento más profundo } \\
\text { de información. }\end{array}$ \\
\hline Aprendizaje & $\begin{array}{l}\text { Procedimientos que el estudiante } \\
\text { adquiere y aplica en forma intencional } \\
\text { para solucionar problemas y demandas } \\
\text { académicas. }\end{array}$ \\
\hline
\end{tabular}

Fuente: Flores et al. (2017)

A su vez, Javaloyes (2016) describe las estrategias de aprendizaje (Tabla 2) en modo de secuencias aplicadas de forma autónoma, dirigidas a una meta y controladas por el estudiante, que resulta en una mejora de su eficiencia, calidad de educación o procesos implicados; mantienen activo el cerebro, favorece la motivación y concentración, optimiza medios y aprovecha mejor su tiempo, estimula el trabajo en equipo, con empleo de herramientas tecnológicas y el uso de actividades dentro y fuera del salón de clases.

De modo que existe la necesidad de desarrollar estrategias que permitan al estudiante conocer el proceso de selección de la información requerida, la relación entre el nuevo conocimiento con sus aprendizajes previos y la transmisión de lo aprendido, con el fin de mejorar las áreas cognitivas de los estudiantes (Salazar, 2018). 
Tabla 2.

Estrategias de Aprendizaje

\begin{tabular}{lll}
\hline Dimensión & Naturaleza & Proceso \\
\hline Volitiva & Autorreguladora & Autorregulación \\
\hline & & Atención \\
\cline { 3 - 3 } Cognitiva & \multirow{2}{*}{ Cognitiva } & Odquisición \\
\cline { 3 - 3 } & & Elaboración \\
\cline { 3 - 3 } & & Recuperación \\
\cline { 2 - 3 } & & Mransferencia \\
\cline { 2 - 3 } & Metacognitiva & Metacognición \\
\hline Afectiva & Motivaciónal \\
\hline
\end{tabular}

Fuente: adaptado de Javaloyes (2016)

De esta manera se puede entender que estrategias de aprendizaje tienen dimensiones que por naturaleza logran un aprendizaje significativo, donde el éxito de la educación está en el uso adecuado de procesos de enseñanza (Hernández, Recalte, y Luna, 2015). Por lo descrito, se requiere de un análisis minucioso que contemple la planeación, ejecución y supervisión del accionar educativo considerando que en su mayor parte el estudiante está acompañado del docente, puesto que es él quien crea la metodología que favorece su enseñanza.

Gutiérrez (2018) considera al conocimiento que tienen los estudiantes como parte fundamental para adaptar una ayuda pedagógica a las características que presentan estos. Por tal motivo, surge la necesidad de desarrollar metodologías que desarrollan un diseño micro curricular que utiliza una aula digital equipada y actualizada, la cual permite interactuar y asimilar de mejor manera el aprendizaje de los estudiantes, de esta manera se motiva al docente a impartir una enseñanza de calidad con equidad académica.

Las estrategias aplicadas a los Ambientes Virtuales de Aprendizaje (AVA) proponen la incorporación de estilos de educación (foros, chats, páginas web, herramientas tecnológicas y académicas) como parte de estos entornos. Estos estilos acompañados de la capacidad métodológica del docente, consiguen la asimilación del conocimiento por parte de los estudiantes (Morales y Pereida, 2017).

El término logros de aprendizaje está estrechamente relacionado con rendimiento académico después de estar sometido a una evaluación donde los docentes las cuantifican mediante la asignación de calificaciones. El desempeño técnico del estudiante refleja de manera objetiva el grado de conocimiento adquirido, el cual según López (2017), gran parte de la capacidad del individuo se forma cuando afronta diferentes estapas de la vida más que por tener un buen desempeño académico.

El análisis de los logros de aprendizaje se cuantifican de acuerdo al Reglamento General a la ley Orgánica de Educación intercultural (LOEI), como se presenta en la (Tabla 4). Los alumnos para aprobar un ciclo académico debe tener un promedio final mínimo de 7 es decir (AAR) en la escala 
(Ministerio de Educación, 2015), acorde con las destrezas y contenidos del módulo de Motores de Combustión Interna emitidos por reglamento (Ministerio de Educación, 2016).

\section{Competencias docentes}

El análisis de Álvarez et al. (2017) resalta la importancia de desarrollar competencias en un ambiente funcional, constructivista y en el marco de la rentabilidad, de igual forma determina estrategias educativas modernas que el profesor debe dominar, tales como la implementación de la tecnología de la información y comunicación (TICs) para formar habilidades de comunicación, análisis, manejo de roles e interpretación de resultados trascendentes.

Pérez (2018) genera tres propuestas didácticas (Tabla 3) que detallan las especificaciones y los equipos necesarios para su implementación sobre la base de la disponibilidad del docente, la metodología didáctica adecuada y la colaboración de la empresa privada para lograr un aprendizaje significativo y de calidad.

En el proceso se conjugan tres factores claves: la necesidad de transmitir el conocimiento en función de los intereses compartidos entre docentes y estudiantes, aplicar dichos conocimientos y tomar como eje las bondades que otorgan las TICs y articular la teoría con elementos prácticos. Para ello el profesor desarrolla la interacción reflexiva con el aspecto funcional de los saberes basándose en la motivación del estudiante por aprender.

Tabla 3.

Propuestas didácticas

\begin{tabular}{ll}
\hline Tipo & Especificaciones/Equipos \\
\hline Aula teórica & Acorde a las necesidades de los alumnos y docentes. \\
\hline Aula TIC's & $\begin{array}{l}\text { Ordenadores, software técnico, internet, simuladores específicos de motores } \\
\text { de combustión inter-na, documentación actualizada. }\end{array}$ \\
\hline $\begin{array}{l}\text { Aula taller práctica y } \\
\text { teórica }\end{array}$ & $\begin{array}{l}\text { Motores estacionarios, simulado-res de tableros didácticos, equi-pos de } \\
\text { comprobación de última tecnología. }\end{array}$ \\
\hline
\end{tabular}

Fuente: adaptado de Pérez (2018)

\section{Metodología}

Para el desarrollo del presente trabajo se establecieron 3 fases: medida del logro académico a estudiantes, encuesta a docentes y análisis de los resultados.

\section{Fase 1}

Consiste en recolectar las calificaciones y cuantificarlas para obtener las calificaciones, en una primera etapa se efectuó las evaluaciones del primer quimestre aplicadas por los docentes de cada asignatura a los estudiantes de tercero bachillerato, en una segunda etapa se repitió el proceso pero esta evaluación se realizó en el segundo quimestre, las dos etapas de la primera fase se realizaron en dos instituciones, en la Unidad Educativa "12 de Noviembre" se evaluó a 43 
estudiantes en la primera y segunda etapa y a 35 alumnos de la Unidad Educativa "Antonio Carrillo Moscoso" también en las dos etapas.

\section{Fase 2}

Inició con la aplicación del instrumento ACRA a 4 docentes de las instituciones, el instrumento cuentó con subescalas que se subdividen a su vez en estrategias que analizan; lo cognitivo a partir de las subescalas de adquisición, codificación y recuperación; lo metacognitiva a través de las subescalas de autoconocimiento, autoplanificación, regulación y autoevaluación; por último, el procesamiento en base a la subescala de: autoinstrucción, auto-control y contra distractores de interacciones sociales, de motivación intrínseca y de escape. Las subescalas de las estrategias están contenidas en ítems que componen el Instrumento (ACRA), la división de estas se puede observar en la Tabla 4.

Tabla 4.

Subescalas ACRA de la prueba

\begin{tabular}{ll}
\hline Subescalas & Ítems \\
\hline Adquisición & $1-7$ \\
\hline Atención & $8-11$ \\
\hline Organización & $12-20$ \\
\hline Elaboración & $21-28$ \\
\hline Recuperación y transferencia & $29-36$ \\
\hline Metacognición y Autorregulación & $37-54$ \\
\hline Motivación & $55-65$ \\
\hline
\end{tabular}

Fuente: Javaloyes (2016)

La fase 2 finaliza con la tabulación de los datos obtenidos por medio de la aplicación del cuestionario ACRA, continuando con el análisis y la interpretación de los resultados.

\section{Fase 3}

Se analizaron los resultados de forma cuantitativa; consistió en realizar un comparativo entre el rendimiento estudiantil registrado y el cuestionario de estrategias por parte de los docentes. Se realizó la prueba de Chi Cuadrado con cuadros de contingencia de ambas variables en términos porcentuales generales, la cual contiene la doble relación entre dos variables contenidas en 2 filas y 2 columnas de una matriz de relación, de tal forma que, considerando que dos valores cualesquiera al relacionarse forman un sola recta de valores que pueden ser tomados, se considera un grado de libertad, es decir $v=1$ veces que se usa la información de la muestra. Para complementar el calculo por convención estadística se utiliza $p=0.05$ que es el nivel de significancia y de probabilidad de encontrar el valor limite o crítico.

Por ultimo, estos valores permiten obtener y comparar el valor del chi cuadrado experimental a través de la Ecuación 1 con el valor tabulado (De La Fuente, 2015). 


$$
x_{\infty p}^{2}=\sum \sum_{j} \frac{\left(n_{y}-e_{\varphi}\right)^{2}}{e_{v}}
$$

Dónde:

$X$ exp: Chi euadrado experimental

$n_{y}$ : Valor frecuencia observado

e.; Valoe frecuencia esperado

\section{Herramienta de Evaluación (ACRA)}

Se entiende como herramienta de evaluación a un instrumento utilizado para determinar los resultados entre dos variables en estudio e incluso para dar a conocer índices de mejoras previstas que sustenten un análisis y apoyen la validez de los resultados en el cumplimiento de los objetivos. Las herramientas de evaluación contienen parámetros que permiten su valoración de manera cualitativa y cuantitativa, estas pueden ser obtenidas a través de técnicas contenidas en estratégias y pueden ser observables y claramente evaluables (Tabla 5). Javaloyes (2016) propone un intrumento de evaluación cuyo estudio se fundamenta en las estrategias de aprendizaje en el aula que toma en cuenta los conceptos de las mismas y su fundamentación histórica y teórica. Este instrumento modelo se denomina Instrumento de Adquisición, Codificación, Recuperación y Apoyo (ACRA) y busca medir las subescalas cognitivas y metacognitiva del accionar educativo.

Tabla 5.

Escalas cualitativas y cuantitativas de rendimiento estudiantil.

\begin{tabular}{ll}
\hline Escala Cualitativa & Escala Cuantitativa \\
\hline $\begin{array}{l}\text { Domina los Aprendizaje Requeridos } \\
\text { (DAR) }\end{array}$ & $9.00-10.00$ \\
\hline $\begin{array}{l}\text { Alcanza los Aprendizajes Requeridos } \\
\text { (AAR) }\end{array}$ & $7.00-8.99$ \\
\hline $\begin{array}{l}\text { Está Próximo a Alcanzar los } \\
\text { aprendizajes requeridos (EPAR) }\end{array}$ & $4.01-6.99$ \\
\hline $\begin{array}{l}\text { No alcanza los aprendizajes } \\
\text { Requeridos (NAAR) }\end{array}$ & $\leq 4$ \\
\hline
\end{tabular}

Fuente: adaptado de Ministerio de Educacion (2015)

La Prueba Chi-Cuadrado es una técnica de uso estadístico muy utilizada para determinar características de entorno y valores de clasificación de muestras en relación con atributos. Esta técnica emplea la evaluación de datos de conteo o frecuencia y el análisis de tablas de contenidos con variables en estudio (Mendivelso y Rodríguez, 2018)principalmente en los anlu00e1lisis de tablas de contingencia ( $r$ x c. El análisis de desempeño académico desarrollado por Muley et al. (2018), determina la importancia de la técnica Chi-Cuadrado como aporte para la evaluación del cumplimiento de objetivos en los que hay una diferencia notable entre sus variables.

Existen pruebas de hipótesis de independencia privada localizada desarrolladas por Gaboardi y Rogers (2018) que determinan la aplicabilidad de la técnica Chi-Cuadrado para análisis, 
donde la hipótesis nula no sea un parámetro único. El valor estadístico resultante de la prueba Chi-Cuadrado se basa en datos generados desde la hipótesis en un máximo de $5 \%$ de su cantidad, esto presenta una ponderación de datos significantes para la determinación del valor representativo crítico.

\section{Resultados}

En primer lugar se presentan las calificaciones de los alumnos de cuatro paralelos transcurridos dos quimestres (Tabla 6), esta muestra los promedios de las dos instituciones, y se puede observar que en la Unidad Educativa "Antonio Carrillo Moscoso" los estudiantes no alcanzan los aprendizajes requeridos durante los dos quimestre; pero con el examen supletorio consiguen alcanzar el nivel mínimo requerido, 7 según la (Tabla 4), para superar el nivel académico, mientras que en la Unidad Educativa "12 de Noviembre" no existen inconvenientes.

Tabla 6.

Promedios de Calificaciones Estudiantes Año Lectivo 2018- 2019

\begin{tabular}{lllll}
\hline & \multicolumn{2}{l}{ U.E. 12 de } & \multicolumn{2}{l}{ U.E. Antonio C. } \\
& Noviembre & \multicolumn{2}{l}{ Moscoso } \\
\hline & Paralelo & Paralelo & Paralelo & Paralelo \\
& A & B & C & D \\
\hline Promedios I Quimestre & 7,21 & 7,54 & 5,53 & 7,16 \\
\hline Promedios II Quimestre & 7,66 & 7,25 & 6,58 & 6,72 \\
\hline Promedios Anuales & 7,44 & 7,39 & 6,00 & 6,94 \\
\hline $\begin{array}{l}\text { Promedios finales } \\
\text { después examen } \\
\text { supletorio }\end{array}$ & 7,52 & 7,51 & 7,09 & 7,37 \\
\hline
\end{tabular}

Fuente: elaboración propia

Se puede apreciar que las calificaciones expresadas en la (Tabla 6) en general alcanzan un nivel básico (AAR), limitándose en aprobar el año lectivo, que influye en el promedio anual y por consiguiente mejora luego del examen supletorio; este resultado indica que el incremento de las estrategias aplicadas en el segundo quimestre y en las clases de recuperación optimiza el proceso de enseñanza-aprendizaje y por ende su mejor rendimiento en el examen supletorio.

Para la fase dos se presenta los resultados obtenidos en cada estrategia de aprendizaje utilizado por los docentes según los porcentajes arrojados en los instrumentos de evaluación aplicados, como lo muestran la Figura 1 para el primer quimestre y la Figura 2 para el segundo quimestre. 


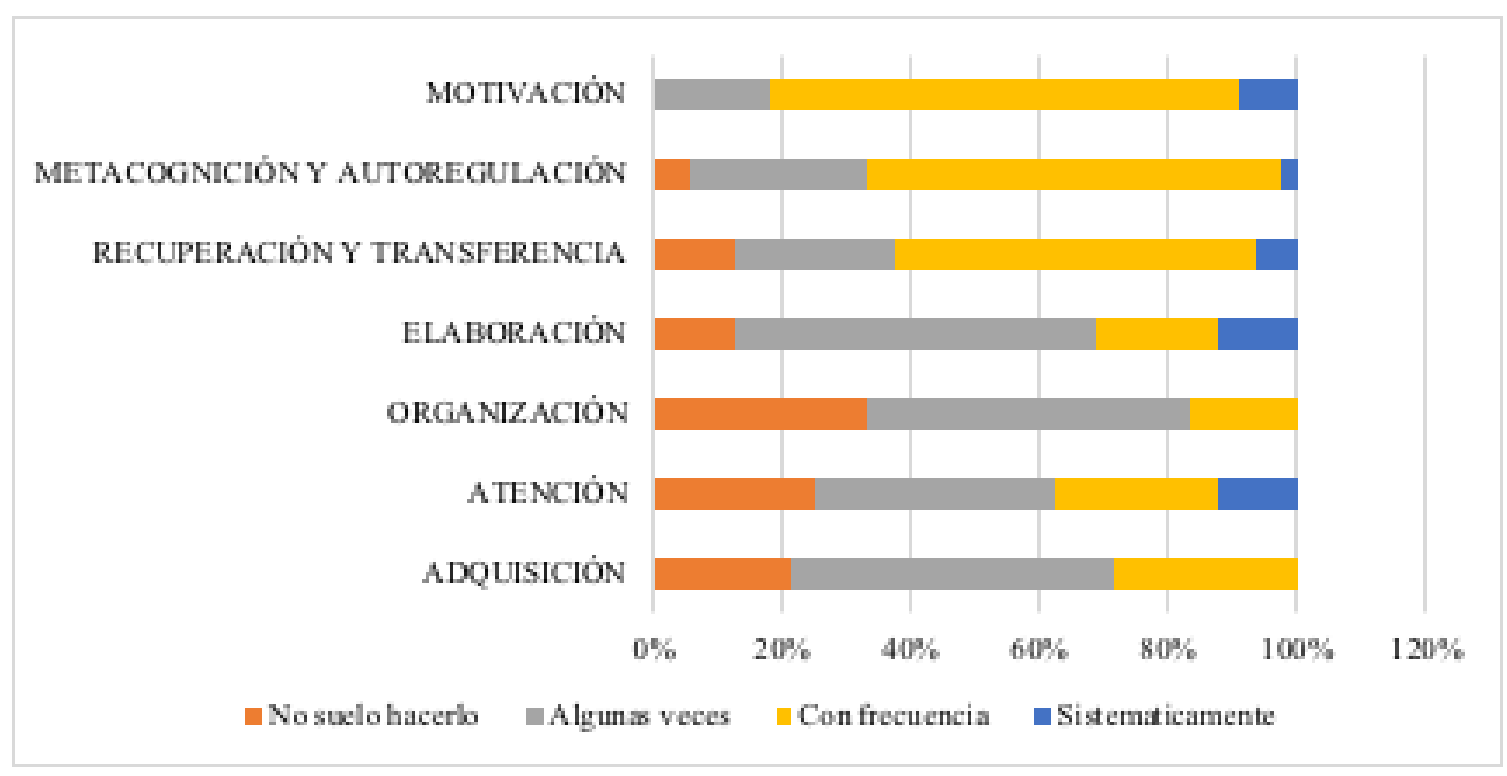

Figura 1. Resultados de Estrategia ACRA aplicada al I Quimestre.

Fuente: elaboración propia

En el primer Quimestre se observa en un alto porcentaje que los docentes no suelen aplicar la estrategia de organización, ni la usan en forma sistemática, únicamente se identifica la estrategia de elaboración según la Tabla 6, puesto que en este quimestre se refleja un bajo rendimiento en el paralelo $\mathrm{C}$, afectando directamente a los estudiantes ya que no les permite aprobar el año lectivo; en el paralelo B se puede observar que el docente debe relacionar los contenidos, esquemas, mapas y tablas para lograr un aprendizaje significativo; es decir, se debe buscar estrategias que optimicen la aplicación de lo estudiado, lo cual es trascendental en el desarrollo de estudiantes de calidad dentro del área. También se puede observar que las estrategias más utilizadas refieren al uso de la motivación seguido por la metacognición y auto-rregulación; dejando como menos utilizada la organización.

La estrategia de metacognición trata de enseñar al estudiante a pensar y ser consciente de su aprendizaje, según los docentes estas estrategias presentan diversas técnicas que apoyan el proceso de enseñanza ya que brindan un valioso aporte para el desarrollo de la asignatura.

Por otro lado, aunque las estrategias de motivación se usan con frecuencia se puede apreciar que la estrategia de atención en el quimestre de adaptación no ocupa un nivel significativo como el de motivación. En definitiva, los resultados infieren en que las estrategias utilizadas y relacionadas a los logros de aprendizaje todavía no permiten mejorar el rendimiento académico, es decir, la atención y la motivación no son elementos suficientes para elevar el nivel de aprendizaje que genera la necesidad de articular estas estrategias con otras más innovadoras. 


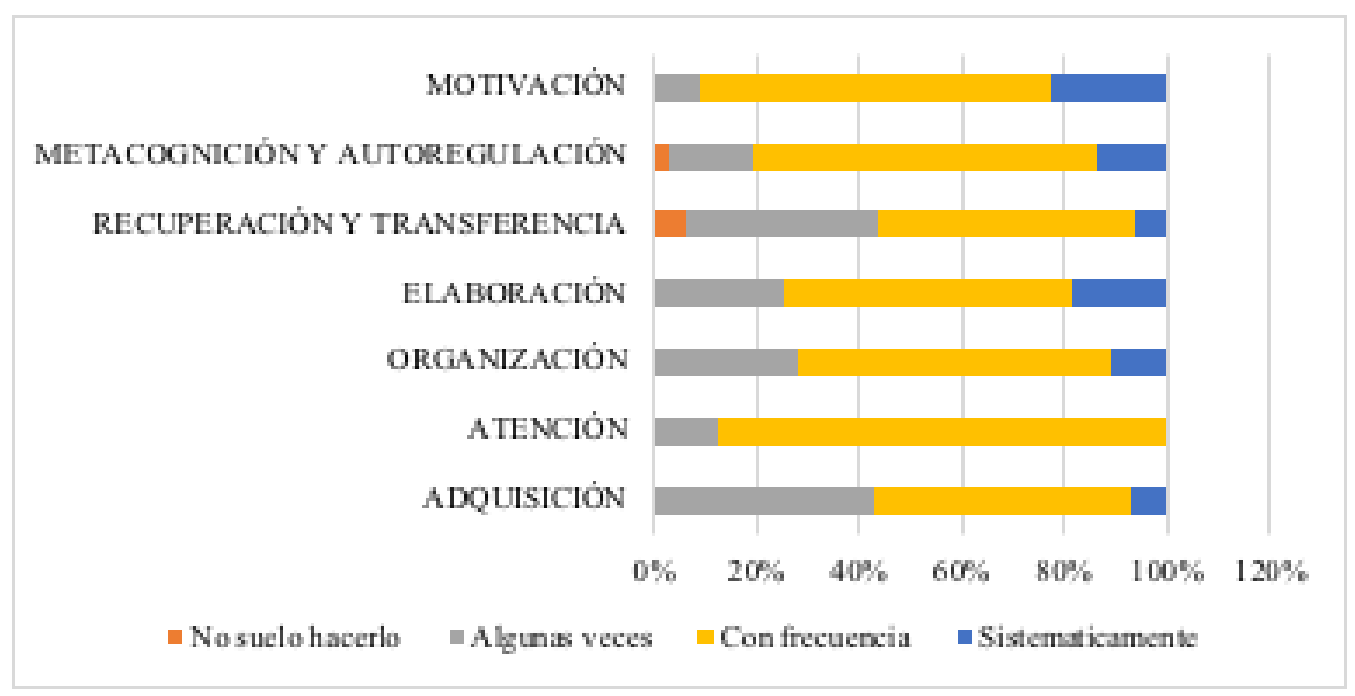

Figura 2. Resultados de Estrategia ACRA aplicada al II Quimestre.

Fuente: elaboración propia

En el segundo quimestre se observa que los docentes incrementan el uso de estrategias, especialmente la motivación, luego la de elaboración; en este caso se observa que la adquisición y la elaboración redujeron su porcentaje, incrementado el rendimiento de los estudiantes en los paralelos $\mathrm{A}$ y $\mathrm{C}$, mientras que en los paralelos $\mathrm{B}$ y $\mathrm{D}$ disminuye; lo que conlleva a deducir que el proceso de enseñanza ha sido significativo, ya que el $100 \%$ de estudiantes lograron aprobar el año lectivo luego de los exámenes finales y supletorios.

Se aprecia también que la organización elevó su porcentaje de un $78 \%$ en el primer quimestre a un $100 \%$ en el segundo quimestre; en el caso de la atención se eleva su nivel de "con frecuencia" en un $25 \%$ del primer quimestre a un $63 \%$ para el siguiente; sin embargo, la metacognición, la autorregulación como la motivación continúan en este período en un nivel alto; donde incluso aumentaron en un 3\% su utilización. Finalmente, se observa que en el segundo quimestre ciertas estrategias redujeron su porcentaje de "no suele hacerlo" y solamente la estrategia de elaboración fue la que menos redujo dicho porcentaje en este mismo parámetro.

Con los resultados obtenidos se establece que la estrategia más viable en el proceso fue la atención, donde se incrementa su uso frecuente aproximadamente tres veces más que el del resto, seguido por el de organización.

Desde el punto de vista de la relación entre las estrategias analisadas en cada una de las Unidades Educativas (Figura 3) se puede identificar las diferencias aplicadas que contibuyeron para que el cumplimiento de los logros de aprendizaje en cada una de ellas sea diferente, lo que causa que la Unidad Educativa "12 de Noviembre" alcanse el nivel requerido mientras la Unidad Educatica "Antonio Carrillo Moscoso" no. De esta análisis se puede determinar que las estrategías que mayor diferencia de aplicación tienen entre las dos Unidades Educativas, con un $25 \%$, son las de; adquisición, atención y elaboración; siendo consideradas como técnicas claves 
que contribuirían a la solucion del inconveniente de logros de aprendizaje en la Unidad Educativa "Antonio Carrillo Moscoso".

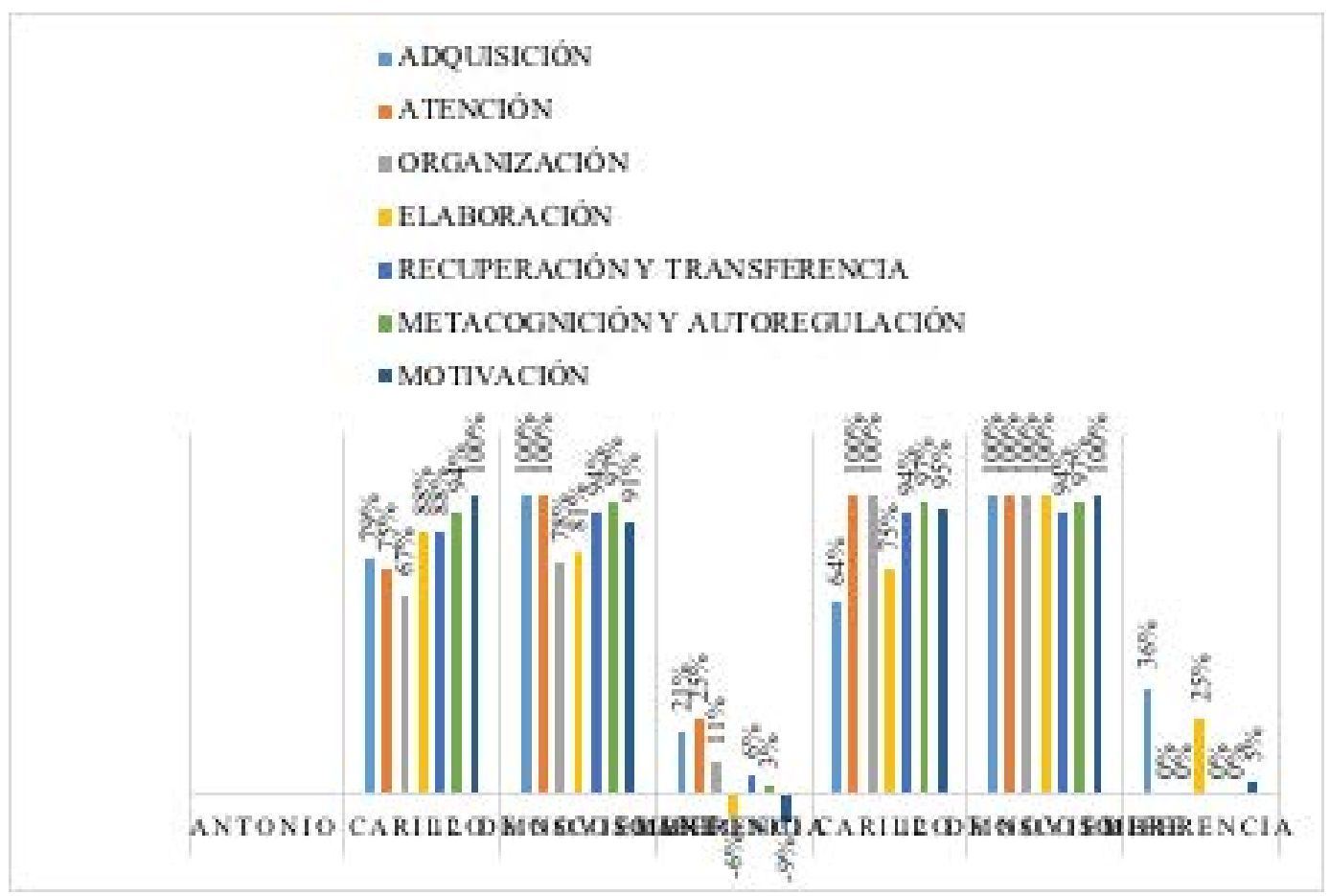

Figura 3. Relación de Estrategias ACRA aplicada a las Unidades Educativas en los dos Quimestre.

Fuente: elaboración propia

Se determinó que todas las estrategias didácticas presentan una asociación entre el rendimiento estudiantil del primer y segundo quimestre con la aplicación de las mismas por parte de los docentes, ya que el valor del chi-cuadrado experi-mental es mayor que el chi-cuadrado tabulado (Tabla 7).

En el período de mayor rendimiento se observa que las estrategias en el aprendizaje son el principal elemento didáctico que el docente debe utilizar, seleccionando adecuadamente cada una de ellas incorporando técnicas apropiadas que direccionen el proceso de enseñanza al uso de la idea previa antes de la lectura, así como la utilización de las palabras claves para recordar información y la organización del testimonio en tablas, secuencias, gráficos, esquemas y relaciones. 
Tabla 7.

Resultados Chi cuadrado ( $\left.X^{2} \exp \right)$

\begin{tabular}{lll}
\hline $\begin{array}{l}\text { Estrategias } \\
\text { didácticas }\end{array}$ & $\begin{array}{l}\text { Chi cuadrado } \\
\text { experimental } \\
(v=1)\end{array}$ & $\begin{array}{l}\text { Chi cuadrado } \\
\text { Tabulado }(v=1 ; \\
\mathrm{p}=0,05)\end{array}$ \\
\hline Adquisición & 33,33 & 3.841 \\
\hline Atención & 225 & 3.841 \\
\hline Organización & 210 & 3.841 \\
\hline Elaboración & 45,33 & 3.841 \\
\hline $\begin{array}{l}\text { Recuperación y } \\
\text { transferencia }\end{array}$ & 50,28 & 3.841 \\
\hline Metacognición & 37,33 & 3.841 \\
\hline Motivacionales & 40,28 & 3.841 \\
\hline
\end{tabular}

Fuente: elaboración propia

En lo que respecta al proceso de repaso de los conceptos importantes y las preguntas que afianza los conocimientos claves. Se señala además que la responsabilidad en el cumplimiento de los plazos de entrega y la consecución de los objetivos pertenece a las estrategias metacognitivas que se encuentran entre las más usada, en cuanto a las estrategias motivacionales, se utilizan las actividades con grupos colaborativos y el refuerzo de la instrucción por encima de las puntuaciones.

Los docentes que obtuvieron mejor resultado implementaron metódicamente a su estrategia el aprendizaje basado en proyectos, el aprendizaje fundamentado en la observación, el estudio basado en la demostración, la gamificación, la enseñanza por descubrimiento y experimentación; lo que indica que los profesores de la Unidad Educativa "Antonio Carrillo Moscoso" deben implementar en sus clases actividades prácticas con el fin de alcanzar mejores logros en la autodidáctica de la asignatura de motores de combustión interna para alcanzar un proceso motivacional que permita obtener bachilleres de calidad dentro de la especialidad.

\section{Conclusiones}

Desde el punto de vista teórico y metodológico, en la investigación se logró establecer que el proceso de enseñanza-aprendizaje depende mucho de las estrategias didácticas que utilizan los profesores de los alumnos de tercer año de Bachillerato en Electromecánica Automotriz de las dos Unidades Educativas comprendidas en el estudio, de las cuales se puede concluir que las estrategias metodológicas más utilizadas por los docentes son la volitiva, cognitiva y afectiva, de esta manera logran un aprendizaje autónomo por parte de los estudiantes.

Se aplicó el cuestionario ACRA con un total de 65 ítems diferentes y siete subescalas con un método correlacional, del cual según los valores de chi-cuadrado experimental y tabulado se determinó que existe una relación significativa entre el uso de todas las estrategias y el rendimiento académico estudiantil. Sobresaliendo las estrategias de atención y organización, seguidas de la metacognición y de motivación. 
El análisis de relación desarrollado entre las estrategias aplicadas permite de manera visual interpretar aquellas estrategias que en mayor o menor proporción son utilizadas para cumplir con el objetivo del logro de aprendizajes en los estudiantes, de esta manera se obtiene un mejor panorama sobre el nivel de consideración y su incidencia en el logro de instrucción de cada Unidad Educativa, logrando de esta manera determinar la importancia de la aplicación de estrategias didácticas en las metodologías empíricas que son usadas por los docentes.

Como punto final, podemos decir que determinar el alcance de cada estrategía es importante para conseguir un sistema de educación óptimo enfocado a logros de aprendizaje, por lo que en trabajos futuros se propondrá la identificación de las mejoras que presenten los estudiantes al modificar el nivel de aplicación de cada estratégia considerada por ACRA para desarrollar sistemas de educación dirigidos o personalizadas. 


\section{Referencias}

Álvarez, J. A., Hernández, R. I., \& Gallegos, M. A. (2017). Implementación de estrategias didácticas basadas en aprendizaje significativo en un curso de circuitos electrónicos. Revista Electrónica ANFEI Digital, 3(6), $1-11$.

De La Fuente, S. (2015). Aplicaciones De La Chi-Cuadrado: Tablas De Contingencia. Homogeneidad. Dependencia E Independencia.

Flores, J., Avila, J., Rojas, C., Sáez, F., Acosta, R., \& Díaz, C. (2017). Estrategias didácticas.

Gaboardi, M., \& Rogers, R. (2018). Local private hypothesis testing: Chi-square tests. 35th International Conference on Machine Learning, ICML 2018, 4(2008), 2625-2645.

García, A., \& Tejedor, F. (2017). Percepción de los Estudiantes Sobre el Valor de las TIC en sus Estrategias de Aprendizaje y su Relación con el Rendimiento. Educación XXI, 20(2), 113-135. https://doi.org/10.5944/ educXX1.1

Gillies, R. M. (2016). Cooperative learning: Review of research and practice. Australian Journal of Teacher Education, 41(3), 39-54. https://doi.org/10.14221/ajte.2016v41n3.3

Gutiérrez, M. (2018). Estilos de aprendizaje, estrategias para enseñar. Su relación con el desarrollo emocional y "aprender a aprender." Tendencias Pedagógicas, 31(2018), 83-96. https://doi.org/10.15366/tp2018.31.004

Hernández, I., Recalte, J., \& Luna, J. (2015). Estrategia Didáctica: Una Competencia Docente En La Formación Para El Mundo Laboral. RIDE Revista Iberoamericana Para La Investigación Y El Desarrollo Educativo, 11(1), 73-94. https://doi.org/10.23913/ride.v9i17.388

Hernandez, R. M. (2017). Impacto de las TIC en la educación: Retos y Perspectivas. Propósitos Y Representaciones, 5(1), 325. https://doi.org/10.20511/pyr2017.v5n1.149

Hoffman, A. F., Ledesma, R., \& Liporace, M. F. (2017). Estilos y estrategias de aprendizaje en estudiantes universitarios de Buenos Aires. Revista de Psicologia (Peru), 35(2), 535-573. https://doi.org/10.18800/ psico.201702.006

Javaloyes, M. J. (2016). Enseñanza De Estrategias De Aprendizaje en el aula.

López, M. (2017). Motivación de logro y rendimiento académico en estudiantes de la facultad de Ingeniería Eléctrica y Electrónica de una Universidad Pública en Lima.

Mendivelso, F., \& Rodríguez, M. (2018). Prueba Chi-Cuadrado de independencia aplicada a tablas 2xN. Revista Médica Sanitas, 21(2), 92-95. https://doi.org/10.26852/01234250.6 
Ministerio de Educación. (2015). Reglamento General a la Ley Orgánica de Educación Intercultural (Issue 4).

Ministerio de Educación. (2016). Guía de contenido para Bachillerato Técnico en Electromecánica automotriz.

Montes De Oca, N., \& Machado, E. (2011). Estrategias docentes y métodos de enseñanza-aprendizaje en la Educación Superior. Humanidades Médicas, 11(3), 475-488.

Muley, A., Bhalchandra, P., Joshi, M., \& Wasnik, P. (2018). Academic analytics implemented for students performance in terms of canonical correlation analysis and chi-square analysis. Advances in Intelligent Systems and Computing, 625, 269-277. https://doi.org/10.1007/978-981-10-5508-9 26

Pérez, J. (2018). Uso pedagógico de los Motores de Combustión Interna Alternativos en la enseñanza del Ciclo Formativo de Grado Medio , especialidad Marítimo Pesquera.

Salazar, C. (2018). Estrategias de enseñanza y aprendizaje para la docencia universitaria. In Enfoque académico (Issue December 2018, pp. 2-21).

Visbal, D., Mendoza, A., \& Díaz, S. (2017). Estrategias de aprendizaje en la educación superior. Sophia-Educación, 13(2), 71-81. 
Copyright (c) 2020 Paco Rubén Alulema y Pablo Israel Amancha Proaño

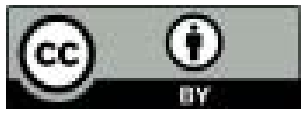

Este texto está protegido bajo una licencia internacional Creative Commons 4.0.

Usted es libre para Compartir-copiar y redistribuir el material en cualquier medio o formato - y Adaptar el documento - remezclar, transformar y crear a partir del material-para cualquier propósito, incluso para fines comerciales, siempre que cumpla las condiciones de Atribución. Usted debe dar crédito a la obra original de manera adecuada, proporcionar un enlace a la licencia, e indicar si se han realizado cambios. Puede hacerlo en cualquier forma razonable, pero no de forma tal que sugiera que tiene el apoyo del licenciante o lo recibe por el uso que hace de la obra.

\section{$\underline{\text { Resumen de licencia - Texto completo de la licencia }}$}

